\title{
ABSENCE OF ABSOLUTELY CONTINUOUS SPECTRUM FOR GENERIC QUASI-PERIODIC SCHRÖDINGER OPERATORS ON THE REAL LINE
}

\author{
DAVID DAMANIK AND DANIEL LENZ
}

\begin{abstract}
We show that a generic quasi-periodic Schrödinger operator in $L^{2}(\mathbb{R})$ has purely singular spectrum. That is, for any minimal translation flow on a finite-dimensional torus, there is a residual set of continuous sampling functions such that for each of these sampling functions, the Schrödinger operator with the resulting potential has empty absolutely continuous spectrum.
\end{abstract}

\section{INTRODUCTION}

In this paper we consider Schrödinger operators

$$
[H \psi](x)=-\psi^{\prime \prime}(x)+V(x) \psi(x)
$$

in $L^{2}(\mathbb{R})$ with quasi-periodic potentials

$$
V(x)=f(\omega+x \alpha) .
$$

Here, $\omega, \alpha \in \mathbb{T}^{d}=\mathbb{R}^{d} / \mathbb{Z}^{d}$ for some $d \in \mathbb{Z}_{+}, f \in C\left(\mathbb{T}^{d}\right)$ real-valued, and $x \in \mathbb{R}$. The case where $V$ is periodic is classical and well understood, and hence we will primarily focus on the aperiodic case. This necessarily means that $d \geq 2$ and it also places some restrictions on $\alpha$ and $f$. We will assume that $\alpha$ is such that the translation flow in question is minimal (i.e., all orbits are dense) to ensure that the torus dimension $d$ is chosen appropriately, and moreover $f$ needs to be non-constant to avoid periodicity.

The spectral properties of operator of the form (1.1) with potentials of the form (1.2) have been studied intensively since the 1980's, with many major advances occurring in the past two decades. Much of this work has been reviewed in several recent survey papers, including [5, 6, 11, 12, 16]. We should point out, however, that some of these survey papers discuss the discrete analogs of these operators, which act in $\ell^{2}(\mathbb{Z})$ as

$$
\left[H^{(d)} \psi\right](n)=\psi(n+1)+\psi(n-1)+V^{(d)}(n) \psi(n)
$$

with

$$
V^{(d)}(n)=f(\omega+n \alpha),
$$

but many results exist in both settings.

Date: September 4, 2019.

D.D. was supported in part by NSF grant DMS-1700131. 
There are, of course, some notable exceptions. One of the most important exceptions is that Avila's global theory for discrete one-frequency quasiperiodic Schrödinger operators with analytic sampling functions [1] does not yet have a continuum counterpart. In this paper we will address another result, which is known in the discrete case, but whose continuum counterpart is desirable to have because of recent progress on the Deift conjecture, which makes a connection with continuum quasi-periodic Schrödinger operators.

The Deift conjecture [7, 8] states that the KdV equation with almost periodic initial data admits global solutions that are almost periodic in (space and) time. The conjecture has been proved under suitable assumptions [4, 9]. These results, and really their proofs, need that the initial data, when considered as potentials, give rise to Schrödinger operators with absolutely continuous spectrum. It was therefore pointed out in [4] that the assumptions will likely fail generically in a suitable sense.

Concretely, the abstract sufficient conditions for the Deift conjecture to hold have been verified for suitable classes of quasi-periodic functions of the form (1.2); see [4]. On the other hand, for discrete quasi-periodic Schrödinger operators, it is known 2 that a generic quasi-periodic potential will give rise to a Schrödinger operator with empty absolutely continuous spectrum. One should therefore expect that also in the continuum case, which is the one relevant to the study of the KdV equation and the Deift conjecture, the absolutely continuous spectrum will be empty for a generic quasi-periodic potential.

The purpose of this paper is to prove this statement:

Theorem 1.1. Given $d \geq 2$ and a minimal translation flow on $\mathbb{T}^{d}, \mathbb{R} \ni$ $x \mapsto \omega+x \alpha \in \mathbb{T}^{d}$, there is a dense $G_{\delta}$-set $\mathcal{S} \subseteq C\left(\mathbb{T}^{d}\right)$ such that for every $f \in \mathcal{S}$, the Schrödinger operator in $L^{2}(\mathbb{R})$ with potential $V(x)=f(\omega+x \alpha)$ has purely singular spectrum.

Remarks 1.2. (a) The minimality of the flow is a property of $\alpha \in \mathbb{T}^{d}$, and the result holds for any such fixed $\alpha$. The set $\mathcal{S}$ will then depend on the choice of $\alpha$.

(b) There is no quantifier on $\omega \in \mathbb{T}^{d}$ in the statement of the result, even though the potential $V$ depends on it. This is due to the constancy of the absolutely continuous spectrum in $\omega$, which is a result of Last and Simon [17. Theorem 1.5].

(c) This result shows that there is a generic obstruction to an extension of the BDGL approach [4] or the EVY approach [9] to the Deift conjecture [7, 8].

One can also consider one-parameter families of potentials and operators by varying the coupling constant:

Theorem 1.3. Given $d \geq 2$ and a minimal translation flow on $\mathbb{T}^{d}, \mathbb{R} \ni$ $x \mapsto \omega+x \alpha \in \mathbb{T}^{d}$, there is a dense $G_{\delta}$-set $\mathcal{S} \subseteq C\left(\mathbb{T}^{d}\right)$ such that for every 
$f \in \mathcal{S}$ and Lebesgue almost every $\lambda>0$, the Schrödinger operator in $L^{2}(\mathbb{R})$ with potential $V(x)=\lambda f(\omega+x \alpha)$ has purely singular spectrum.

\section{Preliminaries}

2.1. Discontinuous Periodic Functions Having Limit-Periodic Limits. Recall that a bounded uniformly continuous function on $\mathbb{R}$ is called almost periodic if for any $\varepsilon>0$ the set of $t \in \mathbb{R}$ with $\|f-f(\cdot-t)\|_{\infty}<\varepsilon$ is relatively dense. A bounded uniformly continuous function on $\mathbb{R}$ is called limit-periodic if it is a uniform limit of continuous periodic functions. But what if we have a uniformly convergent sequence of discontinuous periodic functions? Can the limit be limit-periodic? Clearly, we need to assume at least the continuity of the limit, but what else is needed?

The following statement is likely well known, but since it will play a role in the proof of our main result, we include its short proof for the convenience of the reader.

Proposition 2.1. Suppose $f \in C(\mathbb{R})$ is uniformly continuous and, for $n \geq$ $1, f_{n} \in L^{\infty}(\mathbb{R})$ is periodic. If $\left\|f_{n}-f\right\|_{\infty} \rightarrow 0$ as $n \rightarrow \infty$, then $f$ is limitperiodic.

Proof. The issue is that the $f_{n}$ may be discontinuous and hence the remedy will be to make them continuous via mollification and then to observe that the continuous mollified functions still converge uniformly to $f$. Compare [10. Section C.4] for the definitions and general results below.

Explicitly, define $\eta \in C^{\infty}(\mathbb{R})$ by

$$
\eta(x)= \begin{cases}C \exp \left(\frac{1}{|x|^{2}-1}\right) & \text { if }|x|<1 \\ 0 & \text { if }|x| \geq 1\end{cases}
$$

where $C>0$ is chosen so that $\int_{\mathbb{R}} \eta(x) d x=1$. Then, for $\varepsilon>0$, set

$$
\eta_{\varepsilon}(x)=\frac{1}{\varepsilon} \eta\left(\frac{x}{\varepsilon}\right)
$$

and, for $n \geq 1, f_{n}^{\varepsilon}=\eta_{\varepsilon} * f$, that is,

$$
f_{n}^{\varepsilon}(x)=\int_{\mathbb{R}} \eta_{\varepsilon}(x-y) f_{n}(y) d y .
$$

By the uniform continuity of $f$, Theorem 6 in [10, Section C.4] and its proof (especially the proof of part (iii)), imply that for each $n \geq 1$ and $\varepsilon>0, f_{n}^{\varepsilon}$ is smooth (and in particular continuous) and $\left\|f_{n}^{\varepsilon}-f_{n}\right\|_{\infty} \rightarrow 0$ as $\varepsilon \rightarrow 0$. Thus, the statement follows by diagonalization, that is, for a suitable sequence $\varepsilon_{n} \rightarrow 0$, the functions $f_{n}^{\varepsilon_{n}}$ are continuous, periodic (by construction) and converge uniformly to $f$, showing that $f$ is indeed limitperiodic.

A function $q$ on $\mathbb{R}$ is called eventually periodic if there exists a periodic function $p$ with $p(x)=q(x)$ for all sufficiently large $x \in \mathbb{R}$. 
Corollary 2.2. Suppose $f \in C(\mathbb{R})$ is almost periodic and, for $n \geq 1, f_{n} \in$ $L^{\infty}(\mathbb{R})$ is eventually periodic. If $\left\|f_{n}-f\right\|_{\infty} \rightarrow 0$ as $n \rightarrow \infty$, then $f$ is limit-periodic.

Proof. Let $\varepsilon>0$ be arbitrary. By the preceding proposition it suffices to find a periodic $p \in L^{\infty}(\mathbb{R})$ with $\|f-p\|<\varepsilon$.

By assumption there exists an eventually periodic $q \in L^{\infty}(\mathbb{R})\left(\operatorname{viz} q=f_{m}\right.$ for sufficiently large $m$ ) with

$$
\|f-q\|<\varepsilon .
$$

As $q$ is eventually periodic, there exists a periodic $p \in L^{\infty}(\mathbb{R})$ with $p(x)=$ $q(x)$ for all sufficiently large $x$. Let $P>0$ with $p(x)=p(x+P)$ for all $x \in \mathbb{R}$.

As $f$ is almost periodic, there exists a sequence $\left(t_{n}\right)$ in $\mathbb{R}$ with $\| f_{t_{n}}-$ $f \|_{\infty} \rightarrow 0$ as $n \rightarrow \infty$. Here, we set $g_{t}:=g(\cdot-t)$. There exist then unique $k_{n} \in \mathbb{N}$ and $0 \leq s_{n}<P$ with $t_{n}=k_{n} P+s_{n}$. Restricting attention to a subsequence if necessary, we can then assume without loss of generality that $s_{n} \rightarrow s$. As $f$ is uniformly continuous, we can even assume without loss of generality $s_{n}=s$ for all $n$. To simplify notation we will assume $s=0$.

Hence, $f-p$ is the pointwise limit of $f-q_{t_{n}}$ for $n \rightarrow \infty$. This gives

$$
\begin{aligned}
\|f-p\|_{\infty} & \leq \limsup _{n}\left\|f-q_{t_{n}}\right\|_{\infty} \\
& \leq \limsup _{n}\left(\left\|f-f_{t_{n}}\right\|_{\infty}+\left\|f_{t_{n}}-q_{t_{n}}\right\|_{\infty}\right) \\
& =\left(\lim _{n}\left\|f-f_{t_{n}}\right\|_{\infty}\right)+\limsup _{n}\left\|f_{t_{n}}-q_{t_{n}}\right\|_{\infty} \\
& =\|f-q\|_{\infty} \\
& <\varepsilon .
\end{aligned}
$$

Here, we used the invariance of $\|\cdot\|_{\infty}$ under translation in the penultimate step.

\subsection{Transfer Matrices, Lyapunov Exponents, and Weyl-Titch-} marsh Functions. This subsection recalls important and well-known concepts, mainly to fix notation.

Fixing $d \geq 2$ and a minimal translation flow on $\mathbb{T}^{d}, \mathbb{R} \ni x \mapsto \omega+x \alpha \in \mathbb{T}^{d}$, as well as a real-valued sampling function $f \in C\left(\mathbb{T}^{d}\right)$, the transfer matrices are defined via

$$
\begin{aligned}
\frac{d}{d x} M_{f}(x, E, \omega) & =A_{f}(E, \omega+x \alpha) M_{f}(x, E, \omega) \\
M_{f}(0, E, \omega) & =I
\end{aligned}
$$

for $x \in \mathbb{R}, E \in \mathbb{C}, \omega \in \mathbb{T}^{d}$, where

$$
A_{f}(E, \omega)=\left(\begin{array}{cc}
0 & 1 \\
f(\omega)-E & 0
\end{array}\right) .
$$


These transfer matrices are defined in such a way that $u$ solves the differential equation

$$
-u^{\prime \prime}(x)+f(\omega+x \alpha) u(x)=E u(x)
$$

if and only if it solves

$$
\left(\begin{array}{c}
u(x) \\
u^{\prime}(x)
\end{array}\right)=M_{f}(x, E, \omega)\left(\begin{array}{c}
u(0) \\
u^{\prime}(0)
\end{array}\right) .
$$

By the subadditive ergodic theorem, there is a number $L(E) \geq 0$, called the Lyapunov exponent, so that

$$
L_{f}(E)=\lim _{|x| \rightarrow \infty} \frac{1}{|x|} \log \left\|M_{f}(x, E, \omega)\right\|
$$

for almost every $\omega \in \mathbb{T}^{d}$.

The map $E \mapsto L_{f}(E)$ is real-symmetric and subharmonic. Moreover, we have (see [15, Lemma 3.2 and (49)-(50)])

$$
L_{f}(E)=-\int_{\mathbb{T}^{d}} \operatorname{Re} m_{+, f, \omega}(E) d \omega
$$

for $E \in \mathbb{C}_{+}$, the upper half-plane, where $m_{+, f, \omega}$ is the Weyl-Titchmarsh $m$ function on the right half-line associated with the potential $V(x)=f(\omega+$ $x \alpha$ ), defined by

$$
m_{+, f, \omega}(E)=\frac{u_{+, f, \omega}^{\prime}(0)}{u_{+, f, \omega}(0)},
$$

where $u_{+, f, \omega}$ is a solution of (2.1) that is square-integrable at $+\infty 1$

\section{A Semi-Continuity Result}

In this section we discuss the continuum analog of the Avila-Damanik semi-continuity result [2, Lemma 1]. The general structure of the proof will be the same, and hence we will focus mostly on the aspects that are different between the discrete case and the continuum case.

Set

$$
M_{R}(f)=\operatorname{Leb}\left(\left\{E \in \mathbb{R} \cap[-R, R]: L_{f}(E)=0\right\}\right) .
$$

Remark 3.1. By the Ishii-Kotani-Pastur Theorem [15, Theorem 4.7] and the Last-Simon Theorem [17, Theorem 1.5], we have that $M_{R}(f)=0$ if and only if the Schrödinger operator in $L^{2}(\mathbb{R})$ with potential $V(x)=f(\omega+x \alpha)$ has purely singular spectrum in the energy interval $[-R, R]$ for every $\omega \in \mathbb{T}^{d}$.

Here is the continuum analog of [2, Lemma 1]:

\footnotetext{
${ }^{1}$ To see that such a solution exists, observe that $E \notin \sigma(H)$ by self-adjointness, and hence $\tilde{u}_{+, f, \omega}:=(H-E) \chi_{(-1,0)} \in L^{2}(\mathbb{R})$. But by definition $\tilde{u}_{+, f, \omega}$ solves (2.1) on $(0, \infty)$. Thus, keeping it unchanged on the right half-line and extending it to a solution on $\mathbb{R}$ by solving (2.1), we obtain $u_{+, f, \omega}$.
} 
Lemma 3.2. For all choices of $r, R, \Lambda>0$, the maps

$$
\left(B_{r}\left(L^{\infty}\left(\mathbb{T}^{d}\right)\right),\|\cdot\|_{1}\right) \rightarrow[0, \infty), \quad f \mapsto M_{R}(f)
$$

and

$$
\left(B_{r}\left(L^{\infty}\left(\mathbb{T}^{d}\right)\right),\|\cdot\|_{1}\right) \rightarrow[0, \infty), \quad f \mapsto \int_{0}^{\Lambda} M_{R}(\lambda f) d \lambda
$$

are upper semi-continuous. Here, $B_{r}$ denotes the closed ball with radius $r$ in the essential-supremum norm.

Proof. It is enough to show that (3.1) is upper semi-continuous, the upper semi-continuity of (3.2) then follows from that via Fatou's lemma.

The proof of the upper semi-continuity of (3.1) proceeds in the same way as in [2]. Assuming that the upper semi-continuity of (3.1) fails for some choice of $r, R, \Lambda>0$, there must be $f_{n}, f \in L^{\infty}\left(\mathbb{T}^{d}\right)$ such that

(i) $f_{n} \rightarrow f$ in $L^{1}$ and pointwise as $n \rightarrow \infty$,

(ii) $\left\|f_{n}\right\|_{\infty} \leq r$ for every $n \geq 1$ and $\|f\|_{\infty} \leq r$,

(iii) $\liminf M_{R}\left(f_{n}\right) \geq M_{R}(f)+\varepsilon$ for some $\varepsilon>0$.

By (i) and (ii), we have pointwise convergence of the $m$-functions $m_{+, f, \omega}$ in $\mathbb{C}_{+}$for almost every $\omega \in \mathbb{T}^{d}$ (this follows from a modification of the argument given in [13]). Thus, by (2.2), (ii), and dominated convergence, the associated Lyapunov exponents $L_{f_{n}}$ converge pointwise in $\mathbb{C}_{+}$to $L_{f}$.

Next, consider the region $U$ in $\mathbb{C}_{+}$bounded by the equilateral triangle $T$ with sides $I, J, K$, where $I=[-R, R] \subset \mathbb{R}$. From here the proof proceeds verbatim as in [2, using the Schwarz-Christoffel formula, as well as the fact that the Lyapunov exponent is harmonic in $\mathbb{C}_{+}$and subharmonic (and in particular upper semi-continuous) globally, to derive a contradiction to (iii).

\section{Small Perturbations That Destroy the Absolutely Continuous Spectrum}

In this section we discuss how arbitrarily small perturbations can destroy the absolutely continuous spectrum. Here, we use results of [14.

We first recall some basic concepts from [14]. A piece is a pair $(W, I)$ consisting of an interval $I \subseteq \mathbb{R}$ with length $|I|>0$ (with $|I|=\infty$ allowed) and a locally bounded function $W$ on $\mathbb{R}$ supported on $I$. We abbreviate pieces by $W^{I}$. Without restriction, we may assume that $\min I=0$. A finite piece is a piece of finite length. The concatenation $W^{I}=W_{1}^{I_{1}}\left|W_{2}^{I_{2}}\right| \ldots$ of a finite or countable family $\left(W_{j}^{I_{j}}\right)_{j \in N}$, with $N=\{1,2, \ldots, N\}$ (for $N$ finite) or $N=\mathbb{N}$ (for $N$ infinite), of finite pieces is defined by

$$
I=\left[0, \sum_{j \in N}\left|I_{j}\right|\right],
$$




$$
W=W_{1}+\sum_{j \in N, j \geq 2} W_{j}\left(\cdot-\left(\sum_{k=1}^{j-1}\left|I_{k}\right|\right)\right) .
$$

In this case we say that $W^{I}$ is decomposed by $\left(W_{j}^{I_{j}}\right)_{j \in N}$.

Let now $V$ be a locally bounded function on $\mathbb{R}$. We say that $V$ has the finite decomposition property if there exist a finite set $\mathcal{P}$ of finite pieces and $x_{0} \in \mathbb{R}$ such that $\left(1_{\left[x_{0}, \infty\right)} V\right)$ is a translate of a concatenation $W_{1}^{I_{1}}$ $W_{2}^{I_{2}} \mid \ldots$ with $W_{j}^{I_{j}} \in \mathcal{P}$ for all $j \in \mathbb{N}$. We say that $V$ has the simple finite decomposition property if it has the f.d.p. with a decomposition such that there is $\ell>0$ with the following property: Assume that the two pieces

$W_{-m}^{I_{-m}}|\ldots| W_{0}^{I_{0}}\left|W_{1}^{I_{1}}\right| \ldots \mid W_{m_{1}}^{I_{m_{1}}}$ and $W_{-m}^{I_{-m}}|\ldots| W_{0}^{I_{0}}\left|U_{1}^{J_{1}}\right| \ldots \mid U_{m_{2}}^{J_{m_{2}}}$

occur in the decomposition of $V$ with a common first part $W_{-m}^{I_{-m}}|\ldots| W_{0}^{I_{0}}$ of length at least $\ell$ and such that

$$
1_{[0, \ell)}\left(W_{1}^{I_{1}}|\ldots| W_{m_{1}}^{I_{m_{1}}}\right)=1_{[0, \ell)}\left(U_{1}^{J_{1}}|\ldots| U_{m_{2}}^{J_{m_{2}}}\right)
$$

where $W_{j}^{I_{j}}, U_{k}^{J_{k}}$ are pieces from the decomposition (in particular, all belong to $\mathcal{P}$ and start at 0 ) and the latter two concatenations are of lengths at least $\ell$. Then

$$
W_{1}^{I_{1}}=U_{1}^{J_{1}}
$$

The relevance of the simple finite decomposition property comes from the following result from [18] (see [14] as well).

Lemma 4.1 (Theorem 7.1 of [18]). Let $W$ be a bounded measurable function on $\mathbb{R}$. Assume that both $W$ and $W(-\cdot)$ have the simple finite decomposition property and are not eventually periodic. Then, the Schrödinger operator $H_{W} \psi=-\psi^{\prime \prime}(x)+W(x) \psi(x)$ does not have any absolutely continuous spectrum.

Here is the main result of this section.

Proposition 4.2. Given $d \geq 2$, a minimal translation flow $\mathbb{R} \ni x \mapsto \omega+$ $x \alpha \in \mathbb{T}^{d}, f \in C\left(\mathbb{T}^{d}\right)$, and $\varepsilon>0$, there exists $\tilde{f} \in L^{\infty}\left(\mathbb{T}^{d}\right)$ such that $\| f-$ $\tilde{f} \|_{\infty}<\varepsilon$ and, for all $\omega \in \mathbb{T}^{d}$, the potential $\tilde{V}(x)=\tilde{f}(\omega+x \alpha)$ as well as $\tilde{V}(-\cdot)$ have the simple finite decomposition property and are not eventually periodic. In particular, the Schrödinger operator in $L^{2}(\mathbb{R})$ with potential $\tilde{V}$ has purely singular spectrum.

Proof. It suffices to show the first statement. The last statement then follows from the preceding lemma.

Since the given flow is minimal, we can assume without loss of generality that the function $f$ yields aperiodic potentials $V(x)=f(\omega+x \alpha)$ (otherwise use a fraction of the given $\varepsilon$ to perturb $f$ within $C\left(\mathbb{T}^{d}\right)$ in order to ensure this property). 
For the given $\varepsilon>0$, let us now consider a sequence of partitions $\mathcal{P}_{\varepsilon, n}$ of $\mathbb{T}^{d}$ into finitely many boxes (parallelepipeds) of the following form:

$$
B_{\gamma, \ell}=\left\{\gamma+\sum_{j=1}^{d-1} t_{j} \mathbf{e}_{j}+t_{d} \alpha: 0 \leq t_{j}<\ell_{j} \text { for } 1 \leq j \leq d\right\},
$$

where $\gamma \in \mathbb{T}^{d}$ and $\ell=\left(\ell_{1}, \ldots, \ell_{d}\right)$ with $0<\ell_{1}, \ldots, \ell_{d}<1$. Here $\mathbf{e}_{j}$ denotes the vector that has a 1 as its $j$-th component and only 0's otherwise.

We require two properties from these partitions. These two properties may be satisfied since $f$ is uniformly continuous and the translation flow is minimal. First we ask that for every $n$ and every box $B_{\gamma, \ell}$ belonging to $\mathcal{P}_{\varepsilon, n}$, the variation of $f$ on $B_{\gamma, \ell}$ is less than $\varepsilon / 2$, that is,

$$
\sup _{\omega \in B_{\gamma, \ell}} f(\omega)-\inf _{\omega \in B_{\gamma, \ell}} f(\omega)<\frac{\varepsilon}{2} \text {. }
$$

Second, letting $\delta_{\varepsilon, n}$ denote the maximum of $\|\ell\|_{\infty}$ taken over all boxes $B_{\gamma, \ell}$ in the partition $\mathcal{P}_{\varepsilon, n}$, we require that $\delta_{\varepsilon, n} \rightarrow 0$ as $n \rightarrow \infty$.

Note that once the translation flow enters such a box $B_{\gamma, \ell}$, then it spends exactly $\ell_{d}$ time units in the box before it leaves it again. This is true for each entry into the box, no matter where the entry happens.

Let us now define a function $f_{\varepsilon, n} \in L^{\infty}\left(\mathbb{T}^{d}\right)$ as follows. On each box $B_{\gamma, \ell}$ belonging to $\mathcal{P}_{\varepsilon, n}, f_{\varepsilon, n}$ takes values in the interval

$$
\left[\inf _{\omega \in B_{\gamma, \ell}} f(\omega)-\min \left\{\frac{\varepsilon}{8}, \frac{1}{n}\right\}, \sup _{\omega \in B_{\gamma, \ell}} f(\omega)+\min \left\{\frac{\varepsilon}{8}, \frac{1}{n}\right\}\right],
$$

and moreover the value of $f_{\varepsilon, n}$ at the point $\gamma+\sum_{j=1}^{d-1} t_{j} \mathbf{e}_{j}+t_{d} \alpha$ depends only on $t_{d}$ and is independent of $t_{1}, \ldots, t_{d-1} 2$ Finally we require the dependence of $f_{\varepsilon, n}$ on $t_{d}$ to be continuous and non-constant 3 Such a selection is clearly possible since the interval of allowed values is non-degenerate. Moreover, by construction we have

$$
\left\|f-f_{\varepsilon, n}\right\|_{\infty}<\varepsilon
$$

Now we claim that there is an $n$ so that the statement of the proposition holds for $\tilde{f}:=f_{\varepsilon, n}$. Assume this fails, and we have that in fact for every $n$, the potential $V_{\varepsilon, n}(x)=f_{\varepsilon, n}(\omega+x \alpha)$ or the potential $V_{\varepsilon, n}(-x)$ is eventually periodic or does not have the simple finite decomposition property. Now, clearly, these potentials have the finite decomposition property by construction, and the simplicity of the finite decomposition property of the potential follows by [14, Proposition 3.5] and the local non-constancy aspect of our construction. Thus, for each $n$ the potential $V_{\varepsilon, n}$ or $V_{\varepsilon, n}(-\cdot)$ must be eventually periodic. Restricting attention to a subsequence we can assume without loss of generality that $V_{\varepsilon, n}(x)$ must be eventually periodic

\footnotetext{
${ }^{2}$ This will imply the finite decomposition property below.

${ }^{3}$ We can make it even more regular if needed, such as the function taking any value only a finite number of times. This will then imply the simple finite decomposition property.
} 
for every $n$. Note that the $V_{\varepsilon, n}$ are bounded and measurable, but in general discontinuous. These eventually periodic functions converge (by construction) uniformly to the function $V(x)=f(\omega+x \alpha)$, which is clearly almost periodic, and hence must be limit-periodic due to Corollary 2.2. But since it is manifestly quasi-periodic as well, it must therefore be periodic by 3 , Corollary A.1.4]; contradiction (by our initial step).

Remark 4.3. In the proposition above, once we know that the potential $\tilde{V}(x)=\tilde{f}(\omega+x \alpha)$ and $\tilde{V}(-\cdot)$ have the simple finite decomposition property and are not eventually periodic, these properties are inherited by any nonzero multiple of the potential. In particular it then also follows that, for every $\lambda>0$, the Schrödinger operator in $L^{2}(\mathbb{R})$ with potential $\lambda \tilde{V}$ has purely singular spectrum.

\section{Closing the Jumps}

We saw in Proposition 4.2 that by approximating a given continuous sampling function with a discontinuous sampling function, we can destroy the absolutely continuous spectrum of the associated operator. The approximation is with respect to the $\|\cdot\|_{\infty}$ norm. However, we wish to identify a continuous sampling function that is close to the original one, for which the absolutely continuous spectrum is empty. A second approximation is therefore necessary to close the jumps.

Clearly, the discontinuous function (with the desired property) cannot be approximated by a continuous function in the $\|\cdot\|_{\infty}$ norm. However, it is possible to approximate it in the $\|\cdot\|_{1}$ norm. This shows why the semi-continuity result given by Lemma 3.2 is relevant. Moreover, since the limit function has a zero value and the values are non-negative, the semicontinuity result becomes in effect a continuity result in the setting relevant to this discussion.

The following lemma implements this two-step approximation:

Lemma 5.1. For $f \in C\left(\mathbb{T}^{d}\right)$ and $0<\varepsilon, \delta, R, \Lambda<\infty$, there exists $g \in C\left(\mathbb{T}^{d}\right)$ such that $\|f-g\|_{\infty}<\varepsilon, M_{R}(g)<\delta$, and $\int_{0}^{\Lambda} M_{R}(\lambda g) d \lambda<\delta$.

Proof. Given $f \in C\left(\mathbb{T}^{d}\right)$ and $0<\varepsilon, \delta, R, \Lambda<\infty$, Proposition 4.2 yields an $\tilde{f} \in L^{\infty}\left(\mathbb{T}^{d}\right)$ with $\|f-\tilde{f}\|_{\infty}<\frac{\varepsilon}{2}$ and $M_{R}(\tilde{f})=0$, as well as (cf. Remark 4.3) $M(\lambda \tilde{f})=0$ for every $\lambda>0$.

Let us mollify $\tilde{f}$ (via the mollifiers used in the proof of Proposition 2.1) to produce $f_{n} \in C\left(\mathbb{T}^{d}\right)$ with

$$
\lim _{n \rightarrow \infty}\left\|f_{n}-\tilde{f}\right\|_{1}=0
$$

and

$$
\sup _{n \in \mathbb{Z}_{+}}\left\|f_{n}-f\right\|_{\infty}<\varepsilon
$$


By the non-negativity of the quantities in question, the vanishing limits, and the semi-continuity properties from Lemma 3.2 , it follows that

$$
\lim _{n \rightarrow \infty} M_{R}\left(f_{n}\right)=0
$$

and

$$
\lim _{n \rightarrow \infty} \int_{0}^{\Lambda} M_{R}\left(\lambda f_{n}\right) d \lambda=0 .
$$

Thus, for $n$ large enough, $g=f_{n}$ has the desired properties.

\section{Proof of the Main Results}

In this section we prove the main results, Theorems 1.1 and 1.3 , The proofs are analogous to the corresponding proofs in [2]. Since they are very short, we give the details for the reader's convenience.

Proof of Theorem 1.1. For $0<\delta, R<\infty$, we define

$$
M_{R, \delta}=\left\{f \in C\left(\mathbb{T}^{d}\right): M_{R}(f)<\delta\right\} .
$$

By Lemma 3.2, $M_{R, \delta}$ is open, and by Lemma 5.1, $M_{R, \delta}$ is dense. Thus,

$$
\left\{f \in C\left(\mathbb{T}^{d}\right): \Sigma_{\mathrm{ac}}(f)=\emptyset\right\}=\bigcap_{n \in \mathbb{Z}_{+}} M_{n, \frac{1}{n}}
$$

is a dense $G_{\delta}$ set, as claimed.

Proof of Theorem 1.3. For $0<\delta, R, \Lambda<\infty$, we define

$$
M_{R, \delta}(\Lambda)=\left\{f \in C\left(\mathbb{T}^{d}\right): \int_{0}^{\Lambda} M_{R}(\lambda f) d \lambda<\delta\right\} .
$$

By Lemma 13.2, $M_{R, \delta}(\Lambda)$ is open, and by Lemma [5.1, $M_{R, \delta}(\Lambda)$ is dense. Thus,

$$
\left\{f \in C\left(\mathbb{T}^{d}\right): \Sigma_{\mathrm{ac}}(\lambda f)=\emptyset \text { for a.e. } \lambda>0\right\}=\bigcap_{n \in \mathbb{Z}_{+}} M_{n, \frac{1}{n}}(n)
$$

is a dense $G_{\delta^{-} \text {-set, as claimed. }}$

\section{REFERENCES}

[1] A. Avila, Global theory of one-frequency Schrödinger operators, Acta Math. 215 (2015), 1-54.

[2] A. Avila, D. Damanik, Generic singular spectrum for ergodic Schrödinger operators, Duke Math. J. 130 (2005), 393-400.

[3] J. Avron, B. Simon, Almost periodic Schrödinger operators. I. Limit periodic potentials, Commun. Math. Phys. 82 (1981), 101-120.

[4] I. Binder, D. Damanik, M. Goldstein, M. Lukic, Almost periodicity in time of solutions of the KdV equation, Duke Math. J. 167 (2018), 2633-2678.

[5] D. Damanik, Lyapunov exponents and spectral analysis of ergodic Schrödinger operators: a survey of Kotani theory and its applications, in Spectral Theory and Mathematical Physics: a Festschrift in Honor of Barry Simon's 60th Birthday, 539563, Proc. Sympos. Pure Math. 76, Part 2, Amer. Math. Soc., Providence, RI, 2007. 
[6] D. Damanik, Schrödinger operators with dynamically defined potentials, Ergodic Theory Dynam. Systems 37 (2017), 1681-1764.

[7] P. Deift, Some open problems in random matrix theory and the theory of integrable systems, Integrable Systems and Random Matrices, 419-430, Contemp. Math. 458, Amer. Math. Soc., Providence, RI, 2008.

[8] P. Deift, Some open problems in random matrix theory and the theory of integrable systems. II., SIGMA Symmetry Integrability Geom. Methods Appl. 13 (2017), Paper No. 016, 23 pp.

[9] B. Eichinger, T. VandenBoom, P. Yuditskii, KdV hierarchy via Abelian coverings and operator identities, Trans. Amer. Math. Soc. Ser. B 6 (2019), 1-44.

[10] L. Evans, Partial Differential Equations, Graduate Studies in Mathematics 19, American Mathematical Society, Providence, RI, 1998.

[11] S. Jitomirskaya, Ergodic Schrödinger operators (on one foot), in Spectral Theory and Mathematical Physics: a Festschrift in Honor of Barry Simon's 60th Birthday, 613-647, Proc. Sympos. Pure Math. 76, Part 2, Amer. Math. Soc., Providence, RI, 2007.

[12] S. Jitomirskaya, C. Marx, Dynamics and spectral theory of quasi-periodic Schrödinger type operators, Ergodic Theory Dynam. Systems 37 (2017), 2353-2393.

[13] R. Johnson, J. Moser, Erratum: "The rotation number for almost periodic potentials," Commun. Math. Phys. 90 (1983), 317-318.

[14] S. Klassert, D. Lenz, P. Stollmann, Delone measures of finite local complexity and applications to spectral theory of one-dimensional continuum models of quasicrystals, Discrete Contin. Dyn. Syst. 29 (2011), 1553-1571.

[15] S. Kotani, Generalized Floquet theory for stationary Schrödinger operators in one dimension, Chaos Solitons Fractals 8 (1997), 1817-1854.

[16] S. Kotani, Spectral problems of ergodic Schrödinger operators, Chinese J. Appl. Probab. Statist. 31 (2015), 596-661.

[17] Y. Last, B. Simon, Eigenfunctions, transfer matrices, and absolutely continuous spectrum of one-dimensional Schrödinger operators, Invent. Math. 135 (1999), 329367.

[18] D. Lenz, C. Seifert, P. Stollmann, Zero measure Cantor spectra for continuum onedimensional quasicrystals, J. Differential Equations 256 (2014), 1905-1926.

Department of Mathematics, Rice University, Houston, TX 77005, USA

E-mail address: damanik@rice.edu

Institute for Mathematics, Friedrich-Schiller University, JenA, 07743 JenA

E-mail address: daniel.lenz@uni-jena.de 